\title{
Bottom Communities and Abiotic Factors: Analysis of Statistical Relationship Using the Instability Index and Virtual Species Distribution
}

\author{
Tatiana D. Zinchenko*, \\ Vladimir K. Shitikov and Larisa V. Golovatyuk \\ Samara Federal Research Scientific Center RAS, \\ Institute of Ecology of Volga River Basin RAS \\ Togliatti, Russian Federation
}

\begin{abstract}
Statistical procedures for quantifying the relationships between the community structure and abiotic variables start with selecting a minimum set of uncorrelated environmental factors that determine the ecological conditions essential for each of the species. This is especially important when constructing models of spatial distribution of species which are key to ecology of communities and conservation of nature. The aim of the study is to explore whether some applications of information theory can be used to rank environmental factors with respect to their contribution to the formation of the ecological structure of aquatic communities. We consider the applicability of the instability index, which is a special case of the Kullback-Leibler entropy divergence and reflects the information gain from the displacement of a particular realization of a random variable relative to its mean value. Using of instability indices allows to reduce multidimensional data sets on species structure of communities and abiotic factors to lower dimension sets of commensurate standardized variables and to explore the relationships between the latter. The initial data we used were the results of the long-term (1990-2019) hydrobiological survey of benthic communities in small and medium-sized rivers in the Middle and Lower Volga regions. We consider the indices of instability calculated for each of 147 taxa of macrozoobenthos and 8 geophysical and hydrochemical indicators. Based on these data, we constructed random forest regression models and calculated potential weights of environmental factors that determine ecological preferences of species. The most significant explanatory variables were used to construct distribution maps of «virtual species», which were compared with the corresponding empirical data. A habitat suitability map of chironomids (Diptera, Chironomidae), the Prodiamesinae subfamily, is presented. Instability indices can be effectively used for exploratory analysis of various ecosystems, e. g. ranking
\end{abstract}

(c) Siberian Federal University. All rights reserved

This work is licensed under a Creative Commons Attribution-NonCommercial 4.0 International License (CC BY-NC 4.0).

* Corresponding author E-mail address: zinchenko.tdz@yandex.ru

ORCID: 0000-0002-4435-1166 (Zinchenko T.); 0000-0002-8385-1913 (Shitikov V.); 0000-0003-4773-5277 (Golovatyuk L.) 
habitats according to the degree of environmental instability and / or species associations, selecting the most informative abiotic variables that determine the population density of the taxa, etc.

Keywords: community structure, macrozoobenthos, environmental data, variable selection, Instability index, Kullback-Leibler divergence, virtual species distribution.

Acknowledgements. The authors are grateful to Prof. Cástor Guisande González (University of Vigo, Spain) and the anonymous peer reviewers for their methodological support and invaluable suggestions on improving the manuscript.

The research was carried out in accordance with State Order AAAA-A17-117112040040-3 «Current biodiversity in the ecosystems of the Volga River Basin and its change forecast due to natural and anthropogenic factors» and supported by the Russian Foundation for Basic Research (RFBR) grant 17-04-00135.

\title{
Донные сообщества и абиотические факторы: \\ анализ статистической связи с использованием \\ индекса нестабильности и метода виртуальных видов
}

\author{
Т. Д. Зинченко, В. К. Шитиков, Л. В. Головатюк \\ Самарский федеральный исследовательский центтр РАН \\ Институт экологии Волэсского бассейна РАН \\ Российская Федерация, Тольятти
}

\begin{abstract}
Аннотация. Количественная оценка связи между структурой сообществ и абиотическими переменными предполагает использование статистических процедур, выполняющих селекцию минимального набора некоррелированных факторов среды, которые с высокой вероятностью определяют экологические условия существования каждого вида. Это особенно важно при построении моделей пространственного распределения видов, играющих ключевую роль в экологии сообществ и охране природы. Целью исследования является анализ применения некоторых приложений теории информации для ранжирования факторов среды по их вкладу в формирование экологической структуры сообществ гидробионтов. Рассматривается индекс нестабильности, являющийся частным случаем дивергенции энтропии Кульбака-Лейблера и отражающий информационный выигрыш от смещения конкретной реализации случайной величины относительно ее среднего значения. Использование индексов нестабильности позволяет редуцировать многомерные данные о видовой структуре сообществ и абиотических факторах в наборы соизмеримых стандартизованных переменных и оценить тесноту связи


между ними. В качестве исходных данных используются результаты многолетней (19902019 гг.) гидробиологической съемки донных сообществ малых и средних рек на территории Среднего и Нижнего Поволжья. Обобщены результаты расчетов индексов нестабильности для каждого из 147 таксонов макрозообентоса и 8 геофизических и гидрохимических показателей. На основе этих данных построены регрессионные модели «случайного леса» и рассчитаны веса потенциальной важности факторов среды, определяющих экологические предпочтения видов. Наиболее значимые объясняющие переменные использовались для построения карт распределения «виртуальных видов», которые сравнивались с соответствующими эмпирическими данными. Представлена карта распределения хирономид подсемейства Prodiamesinae (Diptera, Chironomidae) в оптимальных условиях среды обитания. Индексы нестабильности могут эффективно применяться для разведывательного анализа различных экосистем, в том числе ранжирования местообитаний по степени нестабильности окружающей среды и/или видовых ассоциаций, а также отбора наиболее информативных абиотических переменных, определяющих популяционную плотность таксонов.

Ключевые слова: структура сообществ, макрозообентос, факторы среды, отбор переменных, индекс нестабильности, дивергенция Кульбака-Лейблера, распределение виртуальных видов.

Благодарности. Авторы выражают признательность проф. К. Гисанду (University of Vigo, Spain) и двум анонимным рецензентам за методическую поддержку и важные замечания по ходу подготовки рукописи.

Работа выполнена в рамках государственного задания «Оценка современного биоразнообразия и прогноз его изменения для экосистем Волжского бассейна в условиях их природной и антропогенной трансформации» (АAAA-A17-117112040040-3), а также при финансовой поддержке РФФИ (17-04-00135).

Цитирование: Зинченко, Т. Д. Донные сообщества и абиотические факторы: анализ статистической связи с использованием индекса нестабильности и метода виртуальных видов / Т. Д. Зинченко, В. К. Шитиков, Л. В. Головатюк // Журн. Сиб. федер. ун-та. Биология, 2021. 14(2). C. 119-132. DOI: 10.17516/1997-1389-0344

\section{Введение}

Современной парадигмой количественной оценки связи между структурой сообществ и окружающей средой является моделирование пространственно-временного распределения видов в природе (SDM-Species Distribution Models, Peterson et al., 2011). За последние 20 лет этой тематике посвящены сотни публикаций - см. обзоры (Franklin, 2009; Guisan et al., 2017; Лисовский и др., 2020), однако до сих пор в полной мере отсутствует не только единая теория, но и конкретные практические рекомендации. Это обусловлено как объективно существующим многообразием изучаемых экологических сообществ, природно-климатических зон, жизненных форм и техник проведения наблюдений, так и большим арсеналом разработанных методов компьютерной обработки и верификации моделей, выбор которых в значительной мере определяется субъективными взглядами исследователей. В частности, развернутый анализ результатов использования 33 моделей SDM на сообществах птиц, бабочек, дере- 
вьев и травянистой растительности показал (Norberg et al., 2019), что успех моделирования зависит от природы изучаемого сообщества на 36 \%, постановки задачи (интерполяция или экстраполяция) на 26 \%, выбранного алгоритма на 33 \% и объема выборки только на $2 \%$.

Построение моделей SDM требует высокой квалификации аналитика. В частности, в экологических исследованиях для большинства организмов и ситуаций достоверно можно оценить только наличие того или иного вида в точках отбора проб, тогда как надежные данные об «ареалах отсутствия» не могут быть эмпирически обоснованы. Это обусловило разработку различных алгоритмов, основанных на многократном случайном выборе подмножества точек, где, как предполагается, вид отсутствует (pseudo-absence, или «background» points). Из таких алгоритмов можно отметить часто используемый метод максимальной энтропии, реализованный в программе MaxEnt (Phillips et al., 2006). Однако показано (Hastie, Fithian, 2013), что эта процедура оценивает не столько искомую вероятность присутствия вида, сколько неоднородность использованных эмпирических данных, а местоположение и количество точек «псевдо-отсутствия» вида очень зависят от протяженности и характера доступной географической территории. Отмечается также (Лисовский, Дудов, 2020), что работа алгоритма во многом зависит от выбора формы частных функций, предварительной фильтрации исходных данных, использования слоев коррекции и др., а в отсутствие объективных критериев проверки результатов прогноза кажущаяся успешность моделирования может легко ввести в заблуждение.

Перечисленные проблемы побудили разработку математически «простых» процедур, позволяющих выбрать минимальное коли- чество некоррелированных факторов среды, определяющих с высокой вероятностью экологические предпочтения вида, роль которых также может быть обоснована и биологическими причинами. Например, экологический анализ факторов ниши (ENFA; Hirzel et al., 2002) основан на оценке маргинальности $M$ (то есть экологического расстояния между оптимумом вида, полученным из данных его изучения, и средним значением в пределах эталонной области) и специализации $S$ (отношения глобальной дисперсии анализируемого фактора к дисперсии в точках, где обнаружен вид).

Сходный смысл имеет индекс флуктуации Дюбуа (Dubois, 1973), который был в различных вариантах модифицирован под названием индекса нестабильности ${ }^{1}$ I (Guisande et al., 2006, 2017). Применение этого индекса для отбора подмножества информативных предикторов позволяет свести к минимуму влияние коллинеарности на результат любых моделей SDM, но целенаправленно он используется в составе модулей вычислительной среды ModestR (García-Roselló et al., 2014). Этот свободно распространяемый пакет программ обеспечивает комплексную обработку данных о встречаемости видов и абиотических факторов, включая доступ к всемирным базам географической, климатической и ресурсной информации и создание комплекта тематических карт. Включенная в его состав процедура NOO (niche of occurrence - GarcíaRoselló et al., 2019) выполняет интуитивно понятное моделирование вероятностного распределения видов на основе выборочных данных об их присутствии, блокируя попыт-

\footnotetext{
Использование термина “нестабильность” в этом контексте следует признать неудачным, поскольку традиционно в экологии под нестабильностью сообщества или факторов окружающей среды понимается их неустойчивость или изменчивость во времени. Однако прямой перевод Instability index не оставляет иных возможностей.
} 
ки выхода прогнозов за пределы доступной области SDM. Важным компонентом ModestR является база по пресноводным ресурсам мира, на основании которой построены карты распределения рыбных сообществ (GonzálezVilas et al., 2016).

Цель настоящей статьи - анализ результатов апробации индекса нестабильности на примере донных сообществ малых и средних рек в бассейне Средней и Нижней Волги для ранжирования факторов среды и оценки их роли в формировании экологических предпочтений различных таксонов гидробионтов. Наиболее значимые объясняющие переменные использовались для построения карт распределения «виртуальных видов», которые сравнивались с соответствующими эмпирическими данными.

\section{Материал и методика}

Анализ взаимосвязи донных сообществ с факторами среды с использованием индексов нестабильности проводили по результатам многолетних (1990-2019 гг.) исследований на территории Среднего и Нижнего Поволжья (Зинченко, 2009, 2011; Golovatyuk et al., 2018). Гидробиологическую съемку макрозообентоса осуществляли в разные месяцы вегетационного периода на 90 малых и 12 средних равнинных реках, притоках Куйбышевского, Саратовского и Волгоградского водохранилищ, в том числе, на шести реках аридного региона бассейна оз. Эльтон (рис. 1, карта). Средние реки были разделены на приблизительно однородные участки: верхнее, среднее, нижнее течение и устье, а каждая малая река принималась как целостный объект. Таким образом,

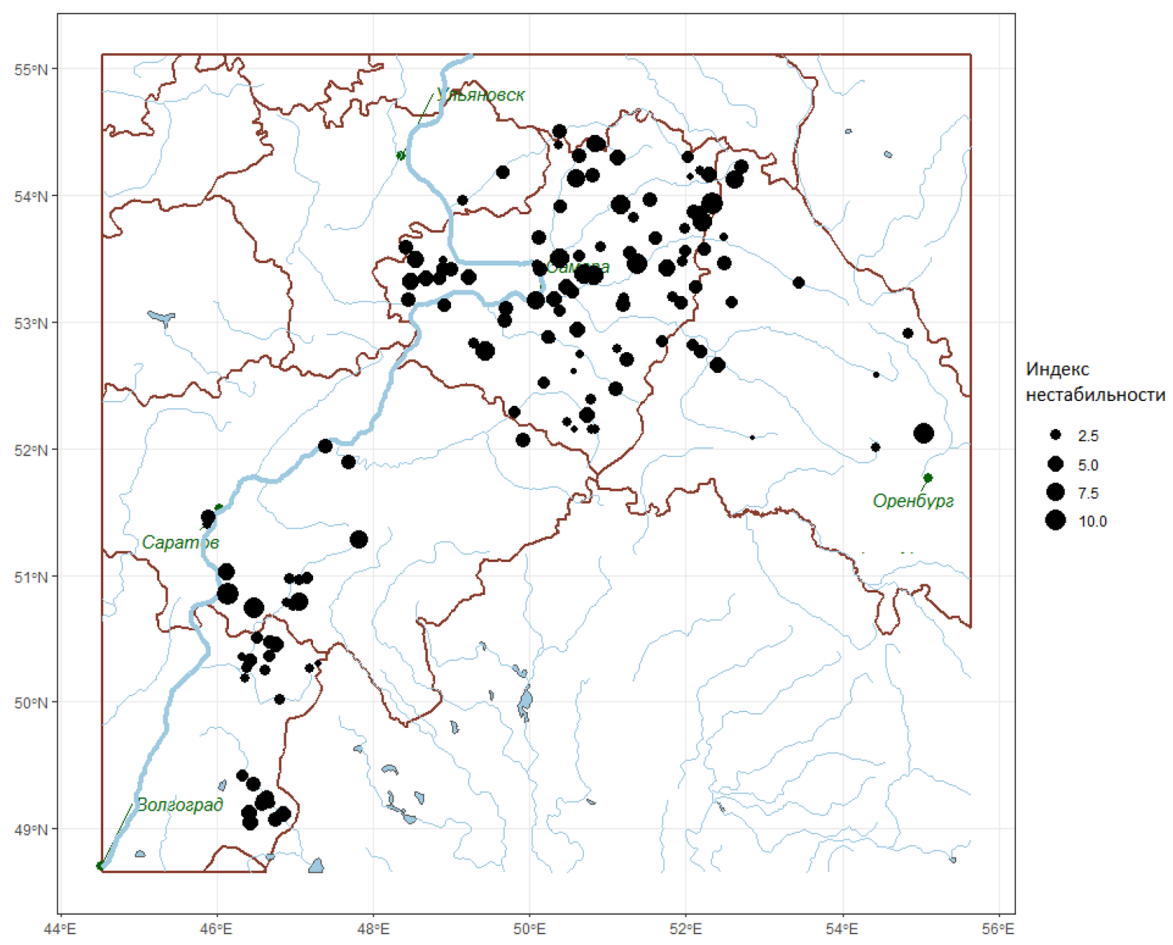

Рис. 1. Карта проведения исследований; размер точек, обозначающих районы проведения гидробиологической съемки, пропорционален индексу нестабильности таксономического состава макрозообентоса

Fig. 1. Map of research area: dots indicate the hydrobiological survey sites; dot size is proportional to the index of instability of macrozoobenthos taxonomic composition

$$
-123-
$$


было исследовано 132 локальных сообщества, в каждом из которых по стандартным методикам выделено до 40 видов макрозообентоса.

Всего было собрано 1400 проб с идентификацией 740 видов и таксонов рангом выше вида, которые для дальнейшего анализа были ограничены списком из 147 видов, встретившихся не менее чем в 15 пробах или на 10 участках из 132. Как единый таксон рассматривалось подсемейство Prodiamesinae в составе трех видов Monodiamesa bathyphila, Odontomesa fulva и Prodiamesa olivacea. Средняя удельная численность (экз/м²) предварительно подвергалась нормирующему преобразованию, приводящему к $\chi^{2}$-дистанции, которое является, по всей вероятности, наиболее разумным компромиссом при учете как роли ведущих компонент, так и вклада редких или малочисленных таксонов (Legendre, Gallagher, 2001). На основе этих данных формировалась матрица TAXA 132×147.

На станциях отбора проб параллельно проводился мониторинг 30 факторов среды, включающих гидрологические параметры водотоков, показатели качества воды и содержание основных химических ингредиентов (состав донных грунтов, насыщение воды кислородом, минерализация и др.). Растровые таблицы, содержащие основные метеорологические и геоморфологические показатели для региона исследований с разрешением 2.5', загружались с серверов свободно распространяемой информации WorldClim и ENVIREM (ENVIronmental Rasters for Ecological Modeling). Поскольку между всеми этими переменными наблюдалась сильная корреляционная связь, проводили анализ индексов инфляции дисперсии VIF (Variance Inflation Factor - Джеймс и др., 2016) и избыточные предикторы со значениями VIF более 30 удаляли из рассмотрения. В результате было отобрано 8 факторов среды, коллинеарность которых считалась приемлемой: среднегодовая температура MTemp, ${ }^{\circ} \mathrm{C}$; осадки самого засушливого квартала PrecDQ, мм; высота Alt, м; индекс шероховатости рельефа TRI; минерализация воды Miner, мг/л; аммонийный азот NH4, мг/л; насыщение кислородом $\mathrm{O}_{2}$, мг/л и категория грунтов Ground в баллах от 1 до 6. На основе этих данных формировалась матрица VAR132×8.

Индекс нестабильности $I_{i j}$ произвольного показателя $x_{i}$ для каждого участка отбора проб $j, j=1 . . . m$, рассчитывали по формуле дивергенции энтропии Кульбака-Лейблера

$$
I_{i j}=p_{i j} \log _{2} p_{i j} / p_{i m},
$$

где $p_{i j}$ - доля $x_{i j}$ в сумме значений этого показателя на всех участках; $p_{i m}-$ среднее $p_{i j}$ для $m$ участков (Guisande et al., 2006). Значение $I_{i j}$ велико при больших отклонениях $x_{i}$ от своего среднего и равняется 0 при $p_{i j}=p_{i m}$. Индексы нестабильности вычислялись как для каждого таксона TAXA, так и каждой переменной VAR, a их суммы $I_{\mathrm{TAXAj}}$ и $I_{\mathrm{VAR} j}$ отражали соответственно нестабильность донного сообщества и факторов окружающей среды, наблюдаемые на участке $j$.

Для оценки связи между подмножествамИ индексов $I_{\mathrm{TAXA}}$ и $I_{\mathrm{VAR}}$ строились модели множественной регрессии методом случайного леса (Random Forest - Шитиков, Мастицкий, 2017), в результате чего для каждого таксона устанавливались величины вклада $W_{i}$ каждого фактора среды, пропорционального их важности (importance). Оценку последней рассчитывали по среднему снижению точности предсказания на оставшихся данных после исключения тестируемого показателя.

Метод распределения «виртуальных видов» (Hirzel at al., 2001) полностью абстрагируется от данных экспедиционных исследований о встречаемости таксонов и моделирует 
ячеистое пространство виртуальной экологической ниши в $n$-мерном пространстве, основываясь исключительно на факторах среды. Для каждой ячейки устанавливается количественно вероятность принадлежности к нише, что фактически является индексом пригодности среды обитания (environmental suitability) для всех видов, у которых экологический оптимум соответствует параметрам априори заданной функции ниши. Для ее определения задавали форму функций отклика для каждого из отобранных абиотических переменных, которые затем обобщались в виде аддитивного или мультипликативного выражения (Leroy at al., 2016).

Расчеты выполнялись с использованием языка статистической среды R3.6. Подробное описание выполненных расчетов и тексты кодов R представлены в файле дополнительных материалов к статье, размещенном на сайте журнала. (http://journal.sfu-kras.ru/ article/141374)

\section{Результаты}

По результатам расчетов получены две матрицы индексов нестабильности: $I_{\text {ТАХА }}$ 132×147 для каждого таксона гидробионтов на каждом участке и $I_{\mathrm{VAR}} 132 \times 8$ для наблюдаемых абиотических переменных. Пространственное распределение суммы индексов $I_{\text {TAхА }}$, рассчитанных по всему их видовому составу, представлено на рис 1.

Строилась модель Random Forest зависимости суммарной нестабильности видов на участках рек от нестабильности абиотических факторов для тех же биотопов и оценивалась относительная важность предикторов. Вклады $W(\%)$, представленные на рис. 2A, дают основание сделать вывод, что флуктуации условий среды в этом регионе, в наибольшей мере влияющие на структуру сообществ, определяются, в основном, статистически «эластичными» показателями, такими как минерализация (Miner), содержание ионов аммония (NH4) и кислорода (O2), а также среднегодовой температурой (MTemp).

Однако 147 частных регрессионных моделей, связывающих подмножества индексов $I_{\mathrm{TAXA}}$ и $I_{\mathrm{VAR}}$ и обобщающих влияние факторов среды на нестабильность численности каждого из видов донных сообществ, обнаруживают более сложную картину. Оценки вклада $W(\%)$ абиотических переменных в вариацию обилия некоторых таксонов макрозообентоса, представленные в табл. 1, показывают, что экологические предпочтения видов чаще всего определяются не одним каким-то фактором, а их различными нестационарными комбинациями. В частности, отдельные виды имеют высокую статистическую зависимость от количества осадков или геоморфологических показателей. В результате обобщения этой информации по всем 147 видам вариация вклада $W$ каждой из абиотических составляющих в изменчивость обилия различных таксонов, имела весьма широкие пределы (рис. 2Б). Если ориентироваться на медианные значения, то структура видового состава донных сообществ по региону определяется, в первую очередь, среднегодовой температурой (МТеmp, $W=22,8)$, минерализацией (Miner, $W=18,9)$ и $\mathrm{NH}_{4}{ }^{+}(W=17,5)$ в отличие от расчлененности рельефа рек (TRI, $W=1,2)$.

Вклады индексов нестабильности предлагается использовать (Guisande et al., 2017) в качестве исходных данных для метода виртуальных видов. В частности, как отражено в табл. 1, численности видов подсемейства Prodiamesinae, обнаруженных на 41 участке рек из 132 обследованных, в наибольшей степени определяются четырьмя геофизическими показателями. Однако для метода виртуальных видов необходимо также указать, каков характер этой связи - прямо или 
A

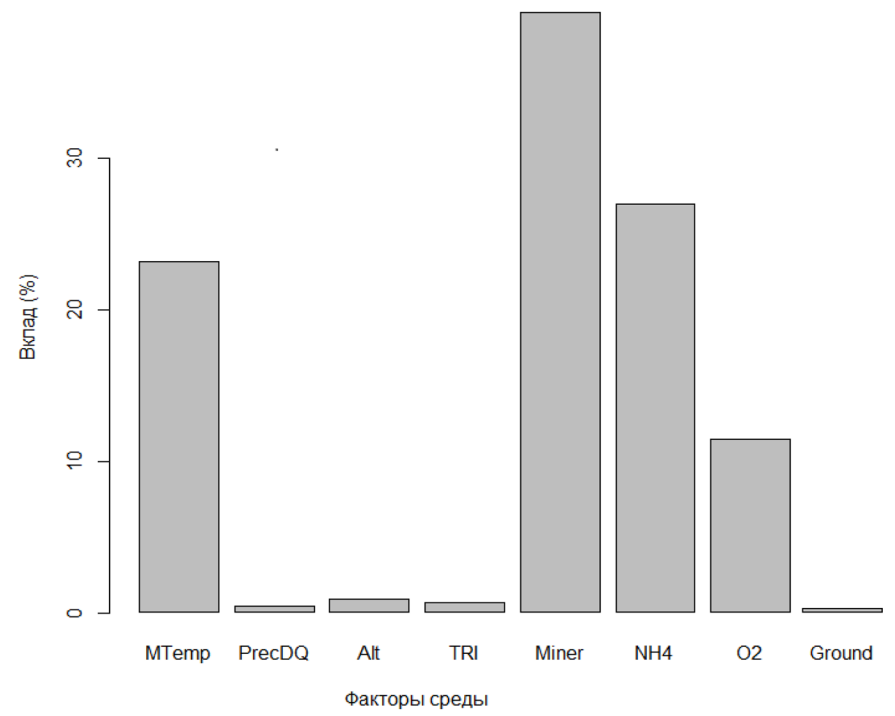

Б

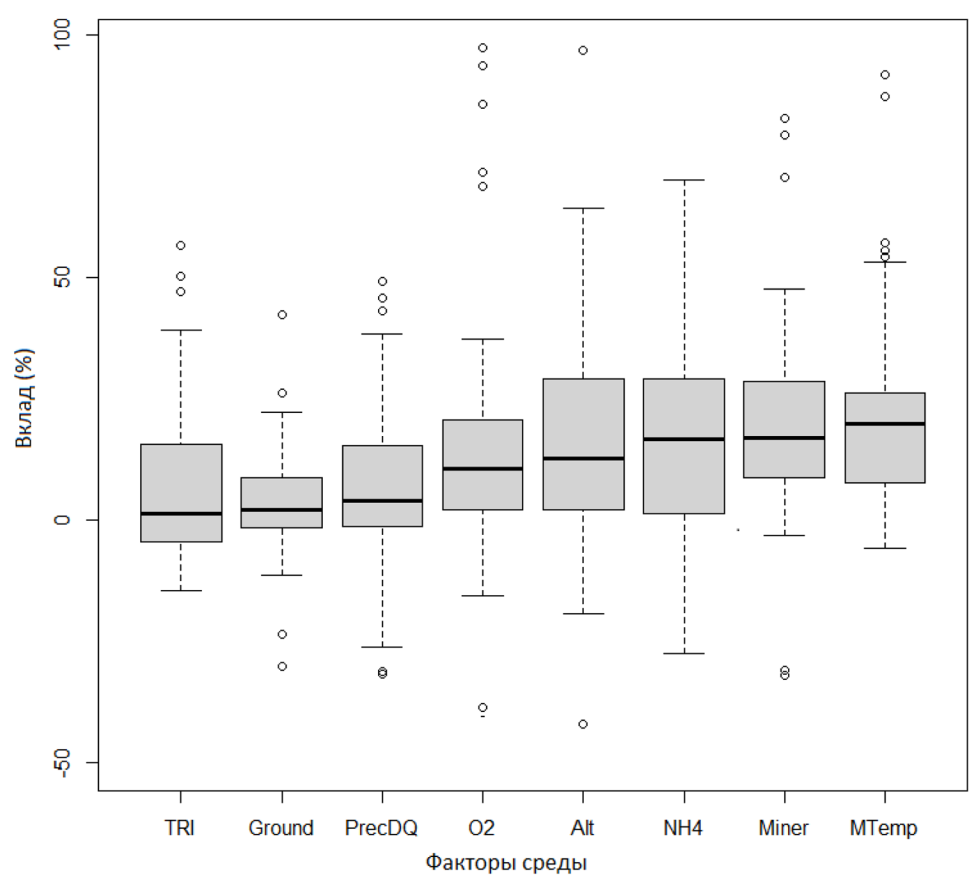

Рис. 2. Вклады отдельных факторов среды в суммарную нестабильность донного сообщества (А) и ранжирование их вариации по отношению к нестабильности каждого из 147 видов (Б). Обозначения показателей: метеорологических - среднегодовая температура (MTemp), осадки самого засушливого квартала (PrecDQ); геоморфологических - высота (Alt), индекс шероховатости рельефа (TRI); гидрохимических - минерализация воды (Miner), аммонийный азот (NH4), насыщение кислородом (O2) и категории грунтов (Ground)

Fig. 2. Shares of environmental factors in total instability of bottom communities (A) and their variation ranked in relation to the instability of each of the 147 species (B). Bars: meteorological factors - average annual temperature (MTemp) and precipitation of the driest quarter (PrecDQ); geomorphological factors - height (Alt) and terrain roughness index (TRI); hydrochemical factors - mineralization of water (Miner), ammonium nitrogen (NH4), oxygen saturation $(\mathrm{O} 2)$ and soil categories (Ground) 
Таблица 1. Оценки вклада $W(\%)$ в нестабильность численности некоторых таксонов макрозообентоса различных факторов окружающей среды ( $m$ - количество проб, в которых встретился вид, из общего числа 1400; обозначения остальных столбцов см. на рис. 2)

Table 1. Estimates of various environmental factors' contribution $(W, \%)$ to population instability for some macrozoobenthos taxa: $m$ - number of samples where the species was found (out of the total of 1400); see Figure 2 for the other columns' names

\begin{tabular}{|c|c|c|c|c|c|c|c|c|c|c|}
\hline Наименование & Подсемейство & $m$ & MTemp & PrecDQ & Alt & TRI & Miner & NH4 & $\mathrm{O} 2$ & Ground \\
\hline Euglesa acuminata & Euglesidae & 27 & 10,82 & 29,98 & 15,02 & 1,65 & 23,10 & 8,05 & 5,20 & 6,18 \\
\hline Pisidium amnicum & Pisidiidae & 33 & 18,89 & 8,68 & 0,29 & 1,77 & 22,99 & 33,85 & 11,04 & 2,50 \\
\hline Ablabesmyia monilis & Tanypodinae & 78 & 21,93 & 3,42 & 11,48 & 2,83 & $-2,06$ & 16,48 & 39,47 & 6,46 \\
\hline Chironomus salinarius & Chironomini & 148 & 32,66 & 12,36 & $-0,46$ & $-1,60$ & 22,39 & 17,84 & 12,62 & 4,18 \\
\hline Cladotanytarsus mancus & Tanytarsini & 323 & 5,57 & 14,02 & $-9,26$ & 16,96 & 19,89 & 32,41 & 13,29 & 7,13 \\
\hline Cricotopus salinophilus & Orthocladiinae & 211 & 21,71 & $-1,83$ & 0,23 & 2,65 & 32,70 & 41,21 & 3,89 & $-0,55$ \\
\hline Dicrotendipes nervosus & Chironomini & 90 & 20,19 & 7,20 & 3,14 & $-0,14$ & 40,89 & 19,45 & 9,22 & 0,06 \\
\hline Paratendipes albimanus & Chironomini & 61 & 33,37 & 0,05 & 51,33 & 3,22 & 6,52 & 2,12 & 3,13 & 0,25 \\
\hline $\begin{array}{l}\text { Polypedilum } \\
\text { nubeculosum }\end{array}$ & Chironomini & 448 & 6,30 & 8,05 & 29,58 & 1,87 & 31,89 & 9,68 & 11,55 & 1,09 \\
\hline Procladius ferrugineus & Tanypodinae & 395 & 22,89 & 15,78 & 11,93 & $-6,85$ & 29,63 & 5,15 & 7,07 & 14,41 \\
\hline $\begin{array}{l}\text { Psectrocladius } \\
\text { sordidellus }\end{array}$ & Orthocladiinae & 75 & 36,88 & 0,42 & 2,97 & $-8,96$ & 17,81 & 26,38 & 21,63 & 2,86 \\
\hline Paratanytarsus confusus & Tanytarsini & 123 & 32,35 & 15,59 & $-2,49$ & $-0,99$ & 13,10 & 18,69 & 23,23 & 0,52 \\
\hline $\begin{array}{l}\text { Stictochironomus } \\
\text { crassiforceps }\end{array}$ & Chironomini & 112 & 37,54 & 12,53 & 2,22 & $-9,19$ & 10,18 & 12,26 & 16,22 & 18,24 \\
\hline Tanypus punctipennis & Tanypodinae & 112 & 21,23 & 13,24 & 18,44 & 27,72 & 3,29 & $-1,40$ & 11,65 & 5,82 \\
\hline Haliplus ruficollis & Haliplidae & 29 & 38,18 & $-3,89$ & 3,21 & $-1,69$ & 13,03 & 29,61 & 18,12 & 3,44 \\
\hline Asellus aquaticus & Asellidae & 86 & 31,19 & 11,31 & $-3,88$ & $-0,56$ & 10,00 & 6,07 & 39,69 & 6,17 \\
\hline Cloeon dipterum & Baetidae & 35 & 46,61 & 5,98 & 25,40 & 12,85 & $-1,88$ & 7,57 & 4,03 & $-0,55$ \\
\hline Caenis robusta & Caenidae & 59 & 48,90 & 6,53 & 10,25 & 15,31 & 7,72 & 5,04 & 4,03 & 2,21 \\
\hline Ephemerella ignita & Ephemerellidae & 32 & $-0,27$ & 56,81 & $-5,96$ & 23,75 & 2,58 & 1,74 & 17,70 & 3,65 \\
\hline Plea minutissima & Pleidae & 27 & 29,99 & 0,51 & 2,17 & 1,21 & 21,07 & 12,56 & 32,53 & $-0,04$ \\
\hline Sialis sordida & Sialidae & 30 & 26,37 & 5,70 & 10,34 & 0,89 & 12,95 & 9,54 & 35,26 & $-1,05$ \\
\hline Gomphus vulgatissimus & Gomphidae & 30 & 21,60 & 6,66 & 20,16 & 0,19 & 8,92 & 25,27 & 17,37 & $-0,19$ \\
\hline Ischnura elegans & Coenagrionidae & 20 & 52,74 & 0,41 & 34,77 & 8,01 & 4,08 & 7,48 & $-7,41$ & $-0,09$ \\
\hline Limnodrilus hoffmeisteri & Tubificidae & 444 & 34,59 & 1,42 & 0,22 & 2,24 & 24,79 & 23,72 & 10,79 & 2,23 \\
\hline Stylaria lacustris & Naididae & 56 & 39,18 & 10,00 & 4,57 & 16,48 & 13,09 & 8,49 & 7,49 & 0,69 \\
\hline Tubifex tubifex & Tubificidae & 272 & 2,95 & $-2,97$ & 9,44 & 21,25 & 28,82 & 9,93 & 9,18 & 21,41 \\
\hline Ecnomus tenellus & Ecnomidae & 61 & 25,14 & 15,18 & 6,92 & 3,81 & 9,73 & 26,28 & 10,48 & 2,45 \\
\hline Prodiamesa sp. & Prodiamesinae & 227 & 21,78 & 18,84 & 36,05 & 8,16 & 6,70 & 1,21 & 1,11 & 6,15 \\
\hline
\end{tabular}

Примечание: отрицательные значения вклада $W$ свидетельствуют об отсутствии влияния фактора (модель в отсутствие этой переменной делает меньше ошибок при прогнозировании). 
обратно пропорциональный, чего ни по индексам нестабильности, ни по оценке их важности установить нельзя. Поэтому по этим предикторам строили модель логистической регрессии, которая имеет вид

$$
\begin{aligned}
& V=-0,069 \text { MTemp }+0,0075 \text { PrecDQ }+ \\
& + \text { 0,0099Alt }+0,0118 \text { TRI, }
\end{aligned}
$$

т.е. вероятность встречаемости продиамезин $V$ прямо зависит от количества летних осадков PrecDQ, высоты Alt и шероховатости рельефа TRI и обратно пропорциональна среднегодовой температуре МТemp. В качестве нишевой функции задавали правую часть уравнения (1) и с использованием метода виртуальных видов и известных геофизических данных по 7280 точкам растра строили карту пригодности среды обитания для продиамезин (и всех других видов с аналогичной зависимостью от абиотических факторов). Затененная часть карты на рис. 3 показывает область с вероятностью встречи этого виртуального таксона более 0,75 . Она охватывает северовосточную часть и правобережье Волги, т. е. более гористую и увлажненную территорию по сравнению с континентальным и степным левобережьем.

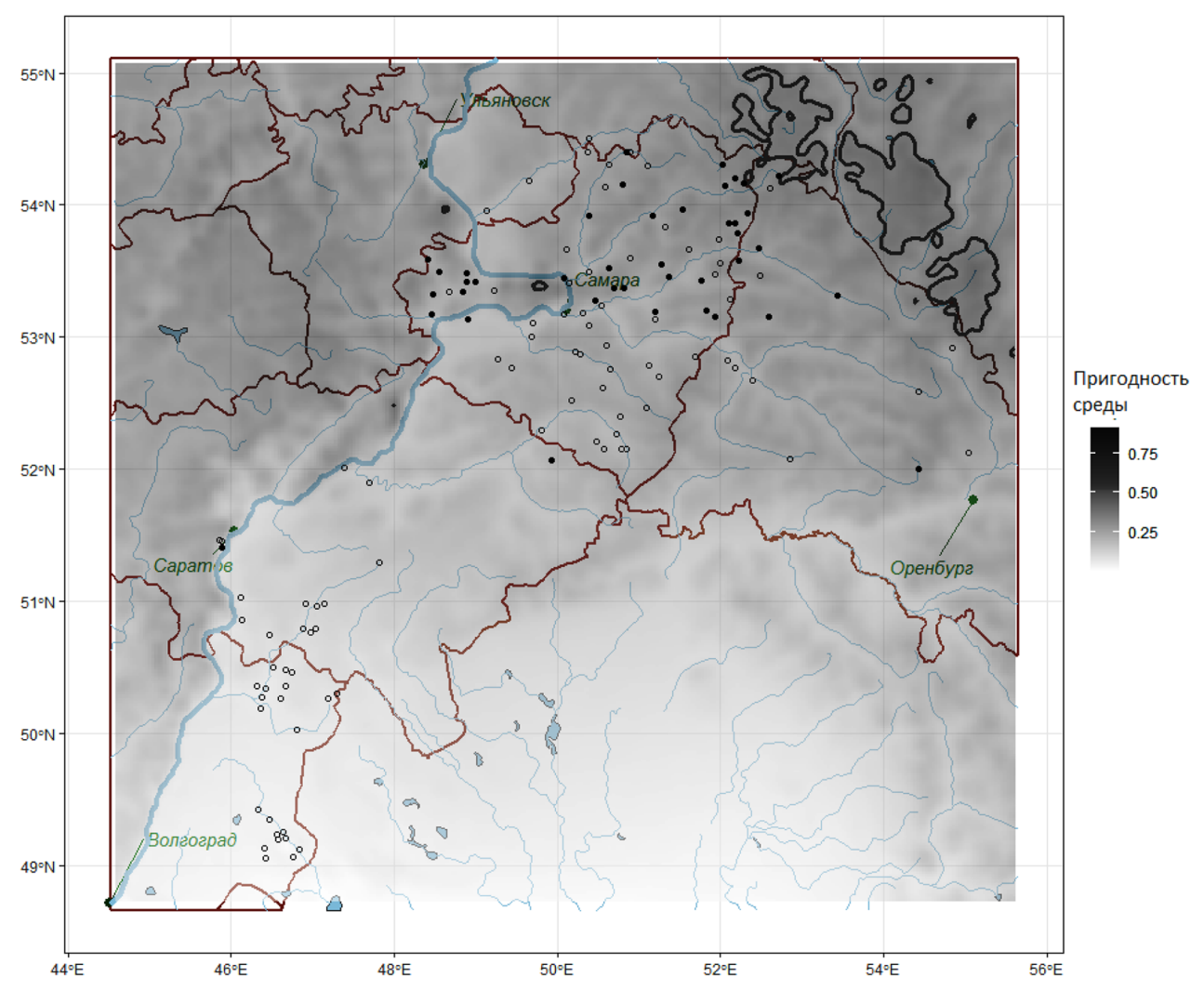

Рис. 3. Пространственное распределение экологических предпочтений Prodiamesinae; контуром отмечена область, где вероятность обнаружения виртуального таксона превышает $0,8 .-$ - точки в районе гидробиологической съемки, где обнаружены виды этого подсемейства, о - отсутствие в пробе

Fig. 3. Habitat suitability map of Prodiamesinae: contoured areas correspond to the probability of finding the virtual subfamily $>0.8$; dots illustrate the hydrobiological survey data $(\bullet-$ sites where the subfamily species were found, $\mathrm{o}-$ sites where the subfamily species were absent) 


\section{Обсуждение}

После появления индекса видового разнообразия Шеннона использование информационных показателей постоянно в фокусе экологии сообществ (Шитиков и др., 2005). Дивергенция Кульбака-Лейблера имеет смысл меры различия энтропии двух эмпирических распределений, а в частном случае индексов нестабильности, отражая тот информационный выигрыш, который исследователь получает, рассматривая конкретную реализацию случайной величины вместо ее выборочного среднего значения. Вычисление сумм $I_{i j}$ для отдельных участков по всем факторам среды или численностям видов в теории информации считается корректным.

Сравнительный анализ индексов нестабильности дает возможность рассмотреть нетривиальные научные гипотезы о роли переменных среды или характерных особенностях таксономической структуры отдельных местообитаний. Моделирование пространственного распределения индексов позволяет выделить в составе изученной территории области со стабильным или нестабильным уровнем популяционной плотности произвольного таксона или величины фактора среды. В рамках геостатистического анализа мы рассматривали наличие тренда, степень автокорреляции и характер функции приращения в зависимости от расстояния между опорными точками. Однако статистически значимых закономерностей распределения точек на рис. 1 обнаружить не удалось. Это приводит к выводу, что значимые однородные кластеры или иные общие тенденции изменчивости структуры сообществ в рамках всего региона не обнаруживаются, что не отрицает существования интересных феноменов в пределах отдельных рек или их локальных сетей.

Как показано выше, величина индекса нестабильности пропорциональна абсолют- ному отклонению показателя в конкретной точке пространства от его среднего значения в пределах всей изучаемой области. При этом остается за рамками характер зависимости, т. е. экстремальным значениям индекса может соответствовать как минимум, так и максимум анализируемого фактора, что часто имеет решающее значение. Поэтому использовать непосредственно индексы нестабильности для построения карт виртуальных видов, как это предложено в статье (Guisande et al., 2017), следует лишь убедившись в одинаковом характере функции связи каждого предиктора с индексом пригодности среды обитания виртуального таксона.

Метод виртуальных видов связывают с понятием экологической ниши - «области в многомерном пространстве всех потенциальных переменных, так или иначе определяющих существование каждого вида и его численность» (G. Hutchinson, цит. по: Пузаченко, 2004, с. 240). Селекция «потенциальных переменных» при наличии необходимого комплекта исходных данных для любого вида или их однородной группы - стандартная, хотя и не простая статистическая процедура. Мы в приведенном примере использовали логистическую регрессию и получили результаты, вполне согласующиеся с биологическими представлениями, что личинки хирономид подсемейства Prodiamesinae являются выраженными рео- и оксибионтами и обитают на каменно-песчаных биотопах проточных рек арктоальпийского типа (Макарченко, Макарченко, 1999). Разумеется, ведущие факторы - высота над уровнем моря и среднегодовая температура - не непосредственно параметры фундаментальной ниши и далеко ими не исчерпываются. Однако они косвенным образом определяют механизмы экологических процессов и характеристики биотопов, а выделенная на рис. 3 область эко- 
логических предпочтений территориально близка к эмпирически построенному ареалу Prodiamesinae, в основном ограниченному реками Бугульминско-Белебеевской возвышенности лесостепной провинции Высокого Заволжья (Зинченко, 2011).

\section{Заключение}

Использование индексов нестабильности в модификации К. Гисанда с соавторами (Guisande et al., 2017) позволяет редуцировать многомерные данные о видовой струк- туре сообществ и абиотических факторов в наборы соизмеримых стандартизованных переменных и оценить тесноту связи между ними. Метод может применяться для предварительного разведывательного анализа компонентов и связей различных экосистем, в том числе для ранжирования местообитаний по степени нестабильности окружающей среды и/или видовых ассоциаций, а также отбора наиболее информативных абиотических переменных, определяющих популяционную плотность таксонов.

\section{Список литературы / References}

Джеймс Г., Уиттон Д., Хасти Т., Тибширани Р. (2016) Введение в статистическое обучение с примерами на языке R. М., ДМК Пресс, 450 с. [James G., Witten D., Hastie T., Tibshirani R. (2017) An introduction to statistical learning (8th ed.). New York, Springer Science+Business Media, 426 p.]

Зинченко Т.Д. (2009) Биоиндикационная роль хирономид (Díptera, Chironomidae) в водных экосистемах: проблемы и перспективы. Успехи современной биологии, 129(3): 257-270 [Zinchenko T.D. (2009) The bioindicative role of Chironomids (Diptera, Chironomidae) in aquatic ecosystems: problems and perspectives. Advances in Modern Biology [Uspekhi sovremennoi biologii], 129(3): 257-270 (in Russian)]

Зинченко Т.Д. (2011) Эколого-фаунистическая характеристика хирономид (Diptera, Chhironomidae) мальх рек бассейна Средней и Нижней Волги (Атлас). Тольятти, Кассандра, 258 c. [Zinchenko T. D. (2011) Ecological and faunal review of chironomids (Diptera, Chironomidae) small rivers in Middle and Low Volga basin (Atlas). Togliatti, Kassandra, 258 p. (in Russian)]

Лисовский А.А., Дудов С.В. (2020) Преимущества и ограничения методов экологического моделирования ареалов. 2. МахEnt. Журнал общей биологии, 81(2): 135-146 [Lissovsky А. А., Dudov S. V. (2020) Advantages and limitations of application of the species distribution modeling methods. 2. MaxEnt. Journal of General Biology [Zhurnal obshchei biologii], 81(2): 135-146 (in Russian)]

Лисовский А.А., Дудов С.В., Оболенская Е.В. (2020) Преимущества и ограничения методов экологического моделирования ареалов. 1. Общие подходы. Журнал общей биологии, 81(2): 123-134 [Lissovsky A. A., Dudov S. V., Obolenskaya E. V. (2020) Advantages and limitations of application of the species distribution modeling methods. 1. A general approach. Journal of General Biology [Zhurnal obshchei biologii], 81(2): 123-134 (in Russian)]

Макарченко Е. А., Макарченко М. А. (1999) Chironomidae. Комары-звонцы. Определитель пресноводных беспозвоночных России и сопредельных территорий. Т. 4. Высшие насекомые. Двукрыльее. СПб., ЗИН РАН, с. 210-296 [Makarchenko Е. А., Makarchenko М. А. (1999) Chironomidae. Ringer mosquitoes. Identification keys for freshwater invertebrates in Russia and adjacent territories. Vol. 4. Higher insects. Dipterous. Saint Petersburg, ZIN RAS, p. 210-296 (in Russian)] 
Пузаченко Ю. Г. (2004) Математические методы в экологических и географических исследованиях. М., Академия, 416 с. [Puzachenko Yu.G. (2004) Mathematical methods in ecological and geographical research. Moscow, Akademiya, 416 p. (in Russian)]

Шитиков В.К., Мастицкий С.Э. (2017) Классификация, регрессия и другие алгоритмы Data Mining с использованием R. Электронная книга: 351 c. URL: https://stok1946.blogspot.com (дата обращения 10.10.2020) [Shitikov V.K., Mastitsky S.E. (2017) Classification, regression and other Data Mining algorithms using R. E-book: 351 p. URL: https://stok1946.blogspot.com (date of access 10.10.2020) (in Russian)]

Шитиков В.К., Розенберг Г.С., Зинченко Т.Д. (2005) Количественная гидроэкология: методы, критерии, решения: в 2-х кн. Москва, Наука, Кн. 1: 281 с.; Кн. 2: 337 с. [Shitikov V. K., Rosenberg G. S., Zinchenko T. D. (2005) Quantitative hydroecology: methods, criteria, solutions: in 2 books. Moscow, Nauka, Book 1: 281 p.; Book 2: 337 p. (in Russian)]

Dubois D.M. (1973) An index of fluctuations, Do, connected with diversity and stability of ecosystems: applications in the Lotka-Volterra model and in an experimental distribution of species. Rapport de sythèse III, Programme National sur l'environment Physique et Biologique, Project Mer. Commision Interministérielle de la Politique Scientifique. Liège

Franklin J. (2009) Mapping species distributions: spatial inference and prediction. Cambridge, Cambridge University Press, 320 p.

García-Roselló E., Guisande C., González-Vilas L., González-Dacosta J., Heine J., PérezCostas E., Lobo J. (2019) A simple method to estimate the probable distribution of species. Ecography, 42(9): $1613-1622$

García-Roselló E., Guisande C., Heine J., Pelayo-Villamil P., Manjarrés-Hernández A., GonzálezVilas L., González-Dacosta J., Vaamonde A., Granado-Lorencio C. (2014) Using ModestR to download, import and clean species distribution records. Methods in Ecology and Evolution, 5(7): 708-713

Golovatyuk L.V., Shitikov V.K., Zinchenko T.D. (2018) Estimation of the zonal distribution of species of bottom communities in lowland rivers of the Middle and Lower Volga basin. Biology Bulletin, 45(10): 1262-1268

González-Vilas L., Guisande C., Vari R., Pelayo-Villamil P., Manjarrés-Hernández A., GarcíaRoselló E., González-Dacosta J., Heine J., Pérez-Costas E., Granado-Lorencio C., Palau-Ibars A., Lobo J.M. (2016) Geospatial data of freshwater habitats for macroecological studies: an example with freshwater fishes. International Journal of Geographical Information Science, 30(1): 126-141

Guisan A., Thuiller W., Zimmermann N.E. (2017) Habitat suitability and distribution models: with applications in $R$. Cambridge, Cambridge University Press, 478 p.

Guisande C., Barreiro A., Maneiro I., Riveiro I., Vergara-Castano A.R., Vaamonde A. (2006) Tratamiento de datos. Madrid, Ediciones Diaz de Santos, 365 p.

Guisande C., Garcia-Rosello E., Heine J., Gonzalez-Dacosta J., Gonzalez-Vilas L., Garcia-Perez B.J., Lobo J. M. (2017) SPEDInstabR: an algorithm based on a fluctuation index for selecting predictors in species distribution modeling. Ecological Informatics, 37: 18-23

Hastie T., Fithian W. (2013) Inference from presence-only data; the ongoing controversy. Ecography, 36(8): 864-867

Hirzel A.H., Helfer V., Metral F. (2001) Assessing habitat-suitability models with a virtual species. Ecological Modelling, 145(2-3): 111-121 
Hirzel A.H., Hausser J., Chessel D., Perrin N. (2002) Ecological-niche factor analysis: how to compute habitat suitability maps without absence data? Ecology, 83(7): 2027-2036

Legendre P., Gallagher E. (2001) Ecologically meaningful transformations for ordination of species data. Oecologia, 129(2): 271-280

Leroy B., Meynard C.N., Bellard C., Courchamp F. (2016) virtualspecies, an R package to generate virtual species distributions. Ecography, 39(6): 599-607

Norberg A., Abrego N., Blanchet F. G., Adler F. R., Anderson B. J., Anttila J., Araújo M. B., Dallas T., Dunson D., Elith J., Foster S. D., Fox R., Franklin J., Godsoe W., Guisan A., O'Hara B., Hill N. A., Holt R.D., Hui F.K.C., Husby M., Kålås J.A., Lehikoinen A., Luoto M., Mod H.K., Newell G., Renner I., Roslin T., Soininen J., Thuiller W., Vanhatalo J., Warton D., White M., Zimmermann N.E., Gravel D., Ovaskainen O. (2019) A comprehensive evaluation of predictive performance of 33 species distribution models at species and community levels. Ecological Monographs, 89(3): e01370

Peterson A.T., Soberón J., Pearson R.G., Anderson R.P., Martínez-Meyer E., Nakamura M., Araújo M. (2011) Ecological niches and geographic distributions (MPB-49). Princeton, Princeton University Press, 328 p.

Phillips S.J., Anderson R.P., Schapire R.E. (2006) Maximum entropy modeling of species geographic distributions. Ecological Modelling, 190(3-4): 231-259 